Understanding the Structure of Cognitive Noise

Jian-Qiao Zhu ${ }^{1}$, Pablo León-Villagrá ${ }^{1}$, Nick Chater $^{2}$, Adam N. Sanborn ${ }^{1}$

${ }^{1}$ Department of Psychology

${ }^{2}$ Warwick Business School

University of Warwick 


\begin{abstract}
Human cognition is fundamentally noisy. While routinely regarded as a nuisance in experimental investigation, the few studies investigating properties of cognitive noise have found surprising structure. A first line of research has shown that inter-response-time distributions are heavy-tailed. That is, response times between subsequent trials usually change only a small amount, but with occasional large changes. A second, separate, line of research has found that participants' estimates and response times both exhibit long-range autocorrelations (i.e., $1 / f$ noise). Thus, each judgment and response time not only depends on its immediate predecessor but also on many previous responses. These two lines of research use different tasks and have distinct theoretical explanations: models that account for heavy-tailed response times do not predict $1 / f$ autocorrelations and vice versa. Here, we find that $1 / f$ noise and heavy-tailed response distributions co-occur in both types of tasks. We also show that a statistical sampling algorithm, developed to deal with patchy environments, generates both heavy-tailed distributions and $1 / f$ noise, suggesting that cognitive noise may be a functional adaptation to dealing with a complex world.
\end{abstract}




\section{Understanding the Structure of Cognitive Noise}

\section{Introduction}

Human behavior is fundamentally noisy across all kinds of judgments and behaviors (Kahneman, Sibony, \& Sunstein, 2021). In empirical research, noise is generally assumed to be a random fluctuation independent of the underlying signal and previous trials, and hence a nuisance variable, which is removed by averaging results or counterbalancing experimental designs. However, studies investigating the properties of noise in human cognition have instead found interesting structure (Gilden, Thornton, \& Mallon, 1995; Rhodes \& Turvey, 2007).

First, while continuous responses are usually assumed to be normally distributed, it has been found that, in free recall tasks, inter-response intervals (IRIs) follow heavy-tailed distributions. Participants asked to recall animal names (see Figure 1A), mostly produced short intervals between retrievals of animal names, but infrequently their retrieval intervals were much longer. These heavy-tailed distributions of retrieval times, $l$, were well described as power laws: $P(l) \sim l^{-\mu}$ with many participants exhibiting tail exponents of $\mu \approx 2$ (Rhodes \& Turvey, 2007). Interestingly, research on animal foraging suggests that the mobility patterns of a wide array of species also exhibit the same exponents, which are mathematically optimal for blind search in environments in which resources are clumped together (Viswanathan et al., 1999). Together, these results suggest that human memory retrieval amounts to foraging in a patchy psychological space (Hills, Jones, \& Todd, 2012).

Second, when participants make repeated estimates of a temporal duration or a spatial magnitude, their responses are not independent, nor only dependent on the last estimate as would be predicted by a random-walk model. Instead, they also depend on long-ago estimates (Gilden et al., 1995). Similar long-range autocorrelations also occur in the response times of many other cognitive tasks such as mental rotation (Gilden, 1997). These correlations are best described as $1 / f$ noise and can be expressed in the frequency domain, $S(f) \sim 1 / f^{\alpha}$, where $f$ is frequency, $S(f)$ is spectral power, and exponents $\alpha \in[0.5,1.5]$ are considered 1/f scaling (Gilden, 1997; Gilden et al., 1995). 
$1 / f$ noise has been explained as the result of complex organisms displaying a self-organized criticality, and it appears to be a signature of active cognitive processing, because it disappears if participants are not asked to do anything more than simply press a button in response to a randomly-occurring target (Gilden et al., 1995).

Heavy-tailed distributions in trial-by-trial changes and $1 / f$ noise in human cognition have been largely studied in isolation, and as a consequence, standard models of $1 / f$ noise do not predict heavy tails and vice-versa. It is not trivial to produce $1 / f$ noise and the most common descriptive model, fractional Brownian motion, predicts a Gaussian distribution of successive changes instead of a heavy-tailed distribution (Mandelbrot \& Van Ness, 1968). Conversely, the most common model of heavy tails in successive changes, the Lévy flight, is a random-walk model that does not produce long-range autocorrelations (Viswanathan et al., 1999).

Here, we investigate whether $1 / f$ noise and heavy-tailed distributions of trial-by-trial changes co-occur in the same experimental task. We ran two experiments with very different tasks: an animal naming task, previously used to show heavy tails (Rhodes \& Turvey, 2007), and a time estimation task, previously used to show $1 / f$ noise (Gilden et al., 1995). In the animal naming experiment, participants were instructed to type animal names as they came to mind, with the only constraint being that successive names needed to be different. In the time estimation experiment, participants were first presented a demonstration of a target time interval $(1 / 3,1$, or 3 seconds) and then were asked to repeatedly reproduce the interval, as if they were drumming (see Figure 1 and Materials and Methods for details).

To determine whether trial-by-trial changes followed a heavy-tailed distribution, we fit $\mu$ following a similar procedure to (Humphries, Weimerskirch, Queiroz, Southall, \& Sims, 2012), with stricter tests showing qualitatively very similar results (see Supplementary Information). To measure the autocorrelation exponent $\alpha$, we fit a line to the windowed, log-binned power spectrum for low frequencies (i.e., less than 0.1) following (Gilden et al., 1995).

In the animal naming experiment, both the pooled data (see Fig 1A) and all 
individual exponents indicate heavy-tailed distributions, replicating (Rhodes \& Turvey, 2007). Congruent with the idea in memory foraging, participants were more likely to report sequential animal names that belonged to the same category (e.g., patch) than other categories. In addition, IRIs were longer when transitioning between categories, and IRIs were correlated with distance in a semantic embedding (see Supplementary Information). Finally and critically, these data also show $1 / f$ noise, both for the pooled results $(\alpha=1.08)$ and for all individual participants.

Our time estimation experiment also found $1 / f$ noise, replicating (Gilden et al., 1995). For the pooled data, $\alpha$ was $1.10,1.45,1.27$ for the $1 / 3 \mathrm{~s}, 1 \mathrm{~s}$, and 3 s conditions, respectively. 21 of 30 participants had exponents in the $1 / f$ range (i.e., $\alpha \in[0.5,1.5]$ ). Importantly, the time estimation experiment also showed heavy-tailed trial-by-trial changes in the pooled data (see Fig 1B), and in 24 of 30 participants. We present evidence that this finding was not due to lapses of attention in the Supplementary Information.

Overall, in both experiments, heavy-tailed trial-by-trial changes and $1 / f$ noise co-occurred, and many individuals exhibited convincing evidence of both effects: all participants in the animal naming experiment and 17 of 30 participants in the time estimation experiment.

\section{Explanations for the structure in cognitive noise}

The co-occurrence of heavy tails and $1 / f$ noise invalidates the most common accounts for each and calls for another explanation. One possible direction is to describe noise using a more complex statistical process, such as Brownian motion in multifractal time, that can, for some parameter settings, produce both features of cognitive noise (Mandelbrot, 2001). However, this account is incomplete as it does not explain how people are able to perform the time estimation task - it is silent on why participants' average estimates tracked the target times $(M=0.36 \mathrm{~s}$ for $1 / 3 \mathrm{~s}, M=1.19 \mathrm{~s}$ for $1 \mathrm{~s}$, and $M=3.50 \mathrm{~s}$ for $3 \mathrm{~s})$. That is, descriptive models offer a very incomplete account of human behavior. 
An interesting alternative that does explain performance casts the mind as an intuitive statistician: the brain creates probabilistic models of an uncertain world and acts according to the prescriptions of these models when taking action (Chater, Tenenbaum, \& Yuille, 2006; Griffiths \& Tenenbaum, 2006). These theories match human behavior in some domains, but have been criticized on the grounds that they are far too complex to be psychologically plausible, and people show systematic deviations from Bayesian statistical models.

Statisticians address the intractability of probabilistic models by using approximations such as sampling algorithms, and it could be that the brain does the same: it utilizes sampling algorithms similar to those used in statistics to approximate otherwise intractable solutions. Sampling approximations are appealing as they show many of the same deviations from exact probabilistic inference that people do (Zhu, Sanborn, \& Chater, 2020), and provide an explanation for neural variability (Orbán, Berkes, Fiser, \& Lengyel, 2016).

There are many types of sampling algorithms and the simplest, drawing independent samples, requires knowing the probability of every hypothesis. This itself is often computationally intractable, leading to the development of a sophisticated family of sampling algorithms called Markov Chain Monte Carlo (MCMC) (Brooks, Gelman, Jones, \& Meng, 2011). MCMC algorithms traverse the space of hypotheses using only local information about the probability distribution. These local transitions mean that successive samples are unavoidably autocorrelated, a necessary evil as these samples generally convey less information than the same number of independent samples do.

The ability to operate with only local information about a probability distribution is the strength of MCMC algorithms, but it introduces weaknesses. Multi-modal probability distributions, those in which there are multiple clusters of high-probability hypotheses which are separated by regions of low-probability hypotheses, are a challenge to these algorithms. Multimodal probability distributions formalize the idea of patchy mental representations, such as those that researchers assume participants use in animal naming experiments (Rhodes \& Turvey, 2007). 
Having only local knowledge, MCMC algorithms are often stuck in one patch and require an impractically large number of iterations before they can visit other isolated modes. As illustrated in Fig 2, this is a problem both for basic MCMC algorithms such as random walk Metropolis (RWM), and more advanced versions such as Hamiltonian Monte Carlo (HMC). To address these weaknesses, statisticians have developed MCMC algorithms that deal better with multimodal representations. One of the first, Metropolis-coupled MCMC $\left(\mathrm{MC}^{3}\right)$, runs multiple $\mathrm{MCMC}$ chains in parallel: while one chain produces the samples, the remaining chains explore the space for isolated modes (Altekar, Dwarkadas, Huelsenbeck, \& Ronquist, 2004). When an exploring chain finds an isolated mode, the sampling chain is likely to swap positions with the exploring chain, and then start sampling from that isolated mode (see Fig. 2 and description in Supplementary Information).

Past work has not compared how well these different MCMC algorithms match human behavior, most likely because they are difficult to distinguish in most experimental data, and time-series data is needed to do so. We investigated RWM, $\mathrm{MC}^{3}$, and HMC to see which, if any, would produce heavy-tailed distributions and $1 / f$ noise. Neither of these properties naturally arise from any of these algorithms, and in particular, $1 / f$ noise will be the result of a mixture of processes with specific scales, and will show a power-law relationship only within a range. There is no reason to expect a priori that the samplers will emit both heavy-tailed changes and $1 / f$ noise.

We assessed the samples generated from the three algorithms while sampling from unimodal Gaussian and Student's t distributions as well as a bimodal mixture of Gaussians, using random algorithmic parameters. The co-occurrence of heavy-tails and $1 / f$ noise proved diagnostic: while more than one algorithm robustly produce either property alone, only $\mathrm{MC}^{3}$ robustly produced both properties (27\% of parameterizations, compared with $4 \%$ and $2 \%$ for RWM and HMC respectively). $\mathrm{MC}^{3}$ was also the most effective at sampling from a bimodal distribution, with its samples showing the lowest deviation for this distribution across a range of parameters (for details, see Supplementary Information). 


\section{Conclusions}

Our finding that $1 / f$ noise and heavy-tailed trial-by-trial changes co-occur provides more information about the structure of the variability in human cognition, and is useful in distinguishing between different accounts of noise. While other accounts can describe the co-occurrence, the success of a sampling algorithm in doing so while accomplishing task goals raises the possibility that noisy responses are the signature of a rational approximation in action, rather than a systematic problem with the brain's hardware.

\section{Methods and Materials}

\section{Animal Naming Experiment}

Participants. Ten native English speakers (6 female and 4 male, aged between 19-25 years) were recruited from the SONA subject pool of the University of Warwick.

Procedure. Participants were asked to type animal names as they came to mind and were explicitly instructed that they could resubmit previous animals, though not consecutively. Once participants had typed an animal they submitted their choice by pressing the enter key. The inter-response interval (IRI) between two consecutive animal names was the duration between last enter pressed and the very next key response. The experiment lasted about 60 minutes or until the participants submitted 1024 animals. Participants received $£ 6$ for participating.

Analyses. IRIs were calculated per-participant for successive submissions. On average it took participants 5.26 seconds $(S D=11.03)$ to submit an animal name. We fitted autocorrelation exponents to the IRI sequences using windowed, log-binning fits Gilden et al. (1995). Per-participant correlation exponents $(\alpha)$ fits ranged from 0.63 to 1.36, $(M=0.99, S D=0.23)$, and all exponents were in the $1 / f$ range. We also obtained per-participant tail exponents $(\mu)$ ranging from 1.29 to $2.61(M=1.80, S D=0.49)$. 


\section{Time Estimation Experiment}

Participants. Another 37 participants (13 male, 23 female, 1 undisclosed gender, aged between 18 and 41) were recruited through the SONA subject pool of University of Warwick.

Procedure. Participants first listened to a sample of the target temporal interval for 60 seconds, presented as computer-generated beeps. Following this familiarization period, participants were instructed to reproduce the beeps (effectively to estimate the target time interval) by pressing the spacebar when they believed the target interval had elapsed. The next trial began as soon as the last one ended, making the task similar to 'drumming' at the rate of the target interval. The experiment was terminated when participants produced 1030 keystrokes or the maximum experimental duration was reached. Participants were paid relative to the maximum duration of experiment, which varied across the three conditions $(6,20$, and 60 mins $)$, receiving $£ 2$, $£ 4$, and $£ 6$ respectively.

Analyses. Of the initial sample, 30 participants completed over 512 time estimates, and their data was analysed in the main text. There were 10 participants for each of three target time interval conditions $(1 / 3 \mathrm{~s}, 1 \mathrm{~s}$, and $3 \mathrm{~s})$. To exclude possible resting periods, we only analysed the time estimates that were less than 3 times the target time interval. Further analyses to exclude possible resting periods are presented in the Supplementary Information. Per-participant correlation exponents $(\alpha)$ ranged from 0.53 to $2.06(M=1.27, S D=0.44)$ and tail exponents $(\mu)$ ranged from 0.87 to $2.67(M=1.60, S D=0.59)$.

\section{Acknowledgements}

We thank Victoria Eshelby for assisting the research. Zhu, León-Villagrá, and Sanborn were supported by the European Research Council (ERC; 817492-SAMPLING). 
Table 1

Proportion of parameterizations scoring as heavy-tailed $(\mu)$, or $1 / f$ noise $(\alpha)$ for the three samplers (out of 1000). In bold, the largest proportions for $\mu, \alpha$ across samplers.

\begin{tabular}{lrrrrrr}
\hline Target & RWM $\alpha$ & RWM $\mu$ & $\mathrm{HMC} \alpha$ & $\mathrm{HMC} \mu$ & $\mathrm{MC}^{3} \alpha$ & $\mathrm{MC}^{3} \mu$ \\
\hline Gaussian & $27 \%$ & $0 \%$ & $5 \%$ & $4 \%$ & $\mathbf{2 7 \%}$ & $\mathbf{3 9 \%}$ \\
Student's t & $23 \%$ & $7 \%$ & $7 \%$ & $33 \%$ & $\mathbf{5 1 \%}$ & $\mathbf{5 0 \%}$ \\
Bimodal & $36 \%$ & $14 \%$ & $9 \%$ & $5 \%$ & $\mathbf{7 9 \%}$ & $\mathbf{3 8 \%}$ \\
\hline
\end{tabular}


A Animal Naming Experiment

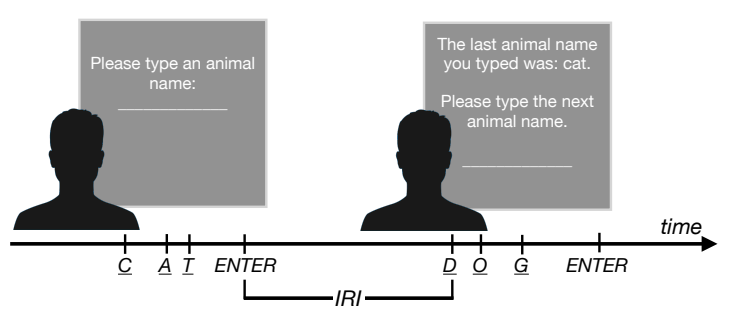

B Time Estimation Experiment

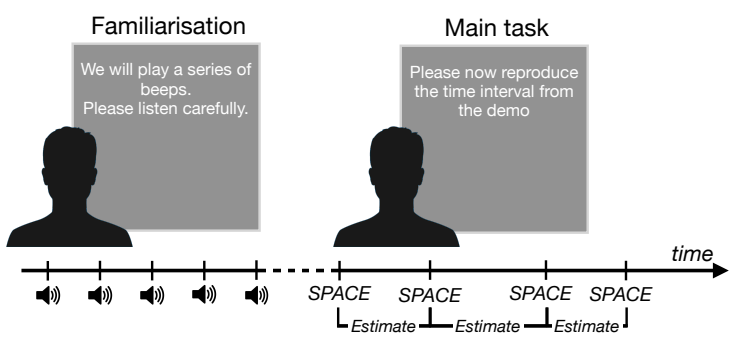

Quantile-quantile Plot

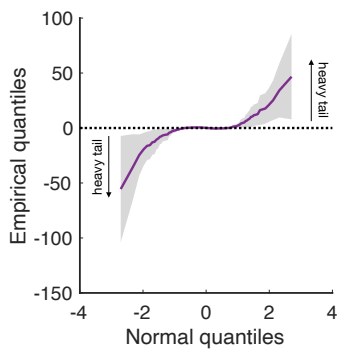

Quantile-quantile Plot

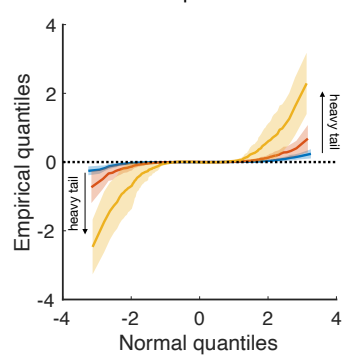

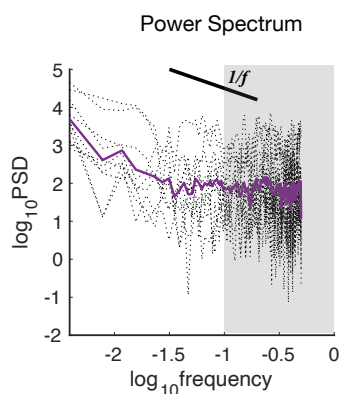

Power Spectrum

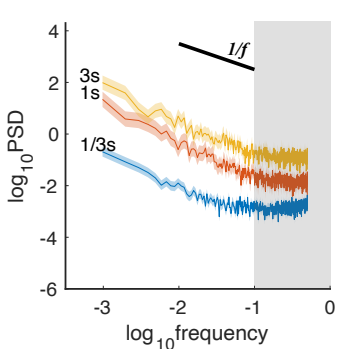

Figure 1. Experiments and results. (A) Animal naming experiment. (B) Time

estimation experiment. Within each row from left to right, there are illustrations of the experimental procedure, quantile-quantile plots of successive changes in IRI or time estimates to test for heavy tails $(95 \%$ CI shaded around means and the horizontal dashed lines denoting the Gaussian distribution), and power spectra to test for $1 / f$ noise (fitted at frequencies less than 0.1, 95\% CI shaded around means). 


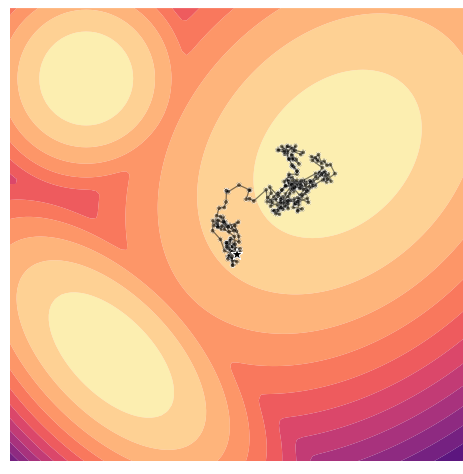

(a) RWM

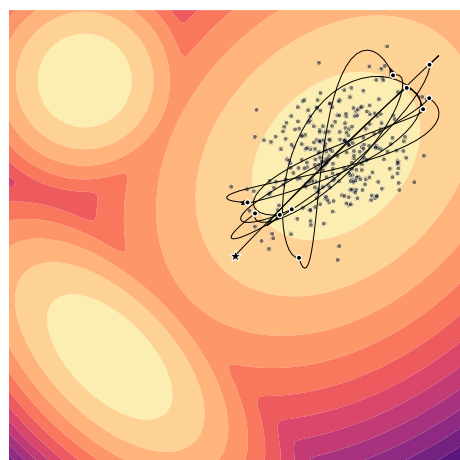

(b) $\mathrm{HMC}$

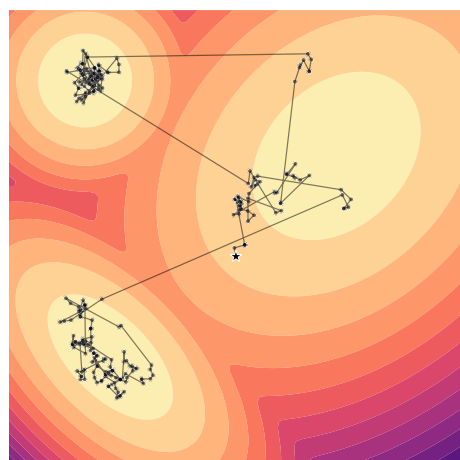

(c) $\mathrm{MC}^{3}$

Figure 2. Example trajectories for the three samplers for a mixture of multivariate Gaussians. (a) Random walk Metropolis (RWM), (b) Hamiltonian Monte Carlo (HMC), and (c) Metropolis-coupled MCMC $\left(\mathrm{MC}^{3}\right)$. 


\section{Appendix A}

\section{Animal Naming Preprocessing and Descriptives}

Submissions were pre-processed by applying spelling corrections, removing punctuation and whitespace characters, removing capitalization and pluralization, and were finally normalized by selecting unified animal names (for instance, "meal worm" as "worm" or "chimp" as "chimpanzee"). The normalization adhered to the animal taxonomy in (Hills et al., 2012) whenever possible. After normalization, a total of 411 unique animal names remained. Participants submitted an average of 148.2 unique animal names $(S D=41.8)$. To assess which categories subsequently named animals belonged to, we applied the category labels used in (Hills et al., 2012; Troyer, Moscovitch, \& Winocur, 1997). Only 79 unique animals in our experiment were not covered by these category labels (139 submissions in total). The first two authors then individually supplied category labels for these remaining animals and devised a categorization scheme consistent with the labels in (Hills et al., 2012; Troyer et al., 1997). After applying these labels, 18 unique animals without a category label remained (48 submissions in total).

We then analyzed the proportion of within-class and between-class transitions for each participant. Transitions were scored as within-class if at least one of the category labels for subsequent animals matched the categories of the previous animal. If no category label existed for an animal, that transition was always scored as between-class. On average, $46.45 \%$ transitions were within class. This proportion was significantly larger than chance $(p<.001$, obtained via 10,000 bootstrapped samples). Transition proportions for individual participants ranged from $34.63 \%-65.31 \%$ $(M=46.87 \%, S D=6.38 \%)$, whereas proportions in bootstrapped independent samples were significantly lower (all $p<.001, M=15.49 \%, S D=4.12 \%$ ). Finally, the duration between successive animals was significantly longer $(t(18)=2.18, p=.04)$ for between-class transitions $(M=6.94 s, S D=14.4)$ than within-class transitions $(M=3.81 s, S D=6.11)$.

To assess whether IRIs correlated with distance in a semantic space, we used the semantic embedding from (Hills et al., 2012) obtained via the BEAGLE model of (Jones 
\& Mewhort, 2007) trained on Wikipedia. We then transformed the cosine similarities between successive animals $x$ and $y$ into euclidean distances via $d(x, y)^{2}=2-2 * \operatorname{sim}(x, y)$. Animals for which no corresponding embeddings were available in (Hills et al., 2012) were dropped from the analysis (139 out of 4967 submissions, $2.8 \%$ ). There was a positive correlation between IRIs and semantic distances, $r=0.14, n=4697, p<.001$. For individual participants we obtained correlations between 0.04 and $0.25(M=0.15, S D=0.07,8$ out of $10 p<.05)$. 
Appendix B

Fitting Methods

Correlation and tail exponents of the time series from both experiments were fitted following conventional methods (Gilden et al., 1995; Humphries et al., 2012). For the correlation exponents $(\alpha)$, we conducted a Fourier analysis using a periodogram function in MATLAB 2020b. The correlation exponent was computed as the negative slope of the fitted line in the windowed, log-binned power spectrum for frequencies less than 0.1, following (Gilden et al., 1995).

For the tail exponents $(\mu)$, we first calculated the distribution of successive changes. The changes are inter-response-intervals and absolute differences between two time estimates in the animal naming experiment and in the time estimation experiment respectively. These distributions were then fitted by a power-law distribution with the exponent and the range of data as free parameters (Humphries et al., 2012). The range was calculated as the part of the dataset with the lowest Kolmogorov-Smirnov (KS) statistic. This was implemented by repeatedly increasing the lower bound and decreasing the upper bound until the KS statistic failed to improve consecutively five times (Humphries et al., 2012). The power-law exponent was then fitted to this subset of the data using maximum-likelihood estimation (Clauset, Shalizi, \& Newman, 2009). In the animal naming experiment, the median fitted range was [2.00s, 61.64s]. In the time estimation experiment, the median fitted ranges were [0.02s, 1.05s], [0.03s, 2.32s], and $[0.01 \mathrm{~s}, 4.10 \mathrm{~s}]$ for $1 / 3 \mathrm{~s}, 1 \mathrm{~s}$, and $3 \mathrm{~s}$ conditions respectively. Using ranges in this way helped exclude resting periods and lapses of attention from the analysis.

In a separate analysis, we looked for a signature of rest periods or lapses of attention in the time estimation experiment: changes in the time estimate that were followed by an immediate reversion to the previous time estimate. In these cases, the ratio of two consecutive changes would be close to -1 . To check whether the apparent heavy tails were due instead to resting periods or inattention, as a conservative test we excluded changes in time estimates associated with change ratios between -1.2 and -0.8 , and reanalyzed the remaining data. Per-participant tail exponents ranged from 0.87 to 
$2.45(M=1.56, S D=0.56)$, and 24 of 30 still were classified as heavy-tailed.

Whether a distribution follows a power law or not is often controversial and technically challenging to establish. To strengthen our results further, we also fitted our data with an alternative, strict approach. As before, we estimated power-law statistics by minimizing the KS-distance of our observed data and the theoretical MLE distribution, as suggested in (Clauset et al., 2009). Instead of the iterative procedure of determining lower- and upper bounds, we fitted a truncated power-law distribution (a power-law distribution times an exponential distribution) with a $x_{m i n}$ truncation. We then contrasted the optimized power-law distribution with the fit of an exponential distribution and report normalized likelihood ratios, as well as bootstrapped $p$-values (Clauset et al., 2009) using the implementation in (Alstott \& Bullmore, 2014). Using this strict test, for all three tapping targets we obtain $\mu$ exponents suggesting power-law distributions $\left(\mu_{1 / 3 s}=1, \mu_{1 s}=1.37, \mu_{3 s}=1.82\right)$. For both 1s and 3s targets, the likelihood ratio strongly and significantly favored the power-law distribution over an exponential $\left(\operatorname{llr}_{1 s}=2.04, p_{1 s}=0.04, \operatorname{ll}_{3 s}=14.87, p_{3 s}<.001\right)$. However, for the aggregate $1 / 3$ s targets the likelihood ratio was not conclusive $\left(\operatorname{llr}_{1 / 3 s}=-.04, p_{1 / 3 s}=.97\right)$. Individual participants' $\mu$ estimates for the $1 / 3$ s target ranged from 1 to $3.9(M=2.64, S D=1.24)$ and eight likelihood ratios favored power-law distributions over exponential, but most ratios were not significant (only $3 / 10 p<.05)$. For the 1 s target, individual participants' estimates ranged from 1 to $3.23(M=1.97, S D=0.78)$ and again eight likelihood ratios favored power-law, but only two likelihood ratios were significant $(2 / 10 p<.05)$. Finally, for the 3 s target, estimates ranged from 1 to $2.9(M=1.58, S D=0.69)$, and nine likelihood ratios favored power-law. In contrast to the $1 / 3$ and 1 s targets, the majority of likelihood ratios were significant $(6 / 10 p<.05)$. For the animal naming data, estimates ranged from 1.5 to $2.59(M=1.51, S D=0.53)$ and all likelihood ratios favored power-law over exponential, with six ratios significantly favoring the power-law $(6 / 10 p<.05)$. Overall, using this strict test, we continued to find evidence for power-law tails in trial-by-trial changes, though in a smaller proportion of participants. 
Appendix C

Sampling Algorithms

The three sampling algorithms discussed in the main text are forms of Markov Chain Monte Carlo (MCMC). While there are elaborate versions of these algorithms with sophisticated tuning schemes that improve their efficiency, we compared simple commonly-used implementations of each.

\section{Random-Walk Metropolis}

Random Walk Metropolis (RWM; see (MacKay, 2003) for an introduction) is a basic and well-known sampling method for sampling from a target distribution $\pi(x)$. It was run with a normal proposal distribution centered on the current state of the sampler (i.e., with zero mean and standard deviation $\sigma$ ).

Given $\sigma$, iterations

Initialize $x_{0}$

for $i=1$ : iterations do

$x_{i} \leftarrow \operatorname{MCMC} \operatorname{STEP}\left(x_{i-1}, \sigma\right)$

end for

function $\operatorname{MCMC} \operatorname{SteP}(x, \pi, \sigma)$

$$
\begin{aligned}
& x^{\prime} \leftarrow x+\mathcal{N}(0, \sigma) \\
& A \leftarrow \min \left\{1, \frac{\pi\left(x^{\prime}\right)}{\pi(x)}\right\} \\
& \text { if } A>u \sim \mathcal{U}_{[0,1]} \text { then } \\
& \quad x \leftarrow x^{\prime}
\end{aligned}
$$$$
\triangleright \text { Propose a move from the current state }
$$

end if

return $x$

end function

\section{Metropolis-coupled Markov Chain Monte Carlo}

Metropolis-coupled Markov Chain Monte Carlo (MC ; see (Altekar et al., 2004) for an introduction) is a more sophisticated sampling algorithm that consists of multiple MCMC chains running at different temperatures. We ran several chains in parallel, with the first chain exploring the untempered distribution, and each successive chain 
exploring an increasingly tempered distribution. While sophisticated temperature schemes have been suggested in the literature, we adopt a linearly spaced temperature scheme, where successive chains explore increasingly tempered distributions.

Given $\pi$, chains, iterations, TEMPERATURE, $\sigma$

$T_{c} \leftarrow$ Temperature $(c)$, for all chains $c$

$\triangleright$ Initialize temperature

Initialize $x_{i=0}^{c}$, for each chain $c$ in $C$ chains

$\triangleright$ Set the initial state of each chain

for $i=1$ : iterations do

for $c=0: C$ do

$x_{i}^{c} \leftarrow \operatorname{MCMC} \operatorname{SteP}\left(x_{i-1}^{c}, \pi^{1 / T_{c}}, \sigma\right) \triangleright$ Perform one MCMC step for the tempered

distribution $\pi$

end for

for $s=0: M / / 2$ do

Select two chains, $k, l, k \neq l$ at random.

$$
\begin{aligned}
& A \leftarrow \min \left\{1, \frac{\pi\left(x_{i}^{k}\right)^{1 / T_{l} \times \pi\left(x_{i}^{l}\right)^{1 / T_{k}}}}{\pi\left(x_{i}^{k}\right)^{1 / T_{k} \times \pi\left(x_{i}^{l}\right)^{1 / T_{l}}}}\right\} \quad \triangleright \text { Metropolis-coupled swapping rule } \\
& \text { if } A>u \sim \mathcal{U}_{[0,1]} \text { then } \\
& x_{i}^{k} \leftarrow x_{i}^{j} ; x_{i}^{j} \leftarrow x_{i}^{k} \quad \triangleright \text { Swap the two chains }
\end{aligned}
$$

end if

end for

end for

\section{Hamiltonian Monte Carlo}

Hamiltonian Monte Carlo (HMC; see (MacKay, 2003) for a conceptual introduction) is an MCMC algorithm that uses Hamiltonian dynamics to make better proposals. We ran HMC with the common leapfrog integration scheme and Gaussian momentum proposals. For pseudocode of the algorithm, see the following Table, where $\epsilon$ is the step-size parameter of the integrator, $\mathcal{L}$ is the log-likelihood of variables $x$, and $L$ is the number of leapfrog integration steps.

\section{Given $\epsilon, L, \mathcal{L}$, iterations}

2: Initialize $x_{0}$

for $i=1$ : iterations do

4: $\quad r^{\prime} \sim \mathcal{N}(0, I)$

$\triangleright$ Propose new momentum 
for $l: L$ do

6:

$$
x^{\prime} \leftarrow \operatorname{LEAPFROG}\left(x, r^{\prime}, \epsilon\right)
$$

end for

8: $\quad A \leftarrow \min \left\{1, \frac{\exp \left(\mathcal{L}\left(x^{\prime}\right)-\frac{1}{2} r^{\prime} \cdot r^{\prime}\right)}{\exp \left(\mathcal{L}(x)-\frac{1}{2} r \cdot r\right)}\right\}$

if $A>u \sim \mathcal{U}_{[0,1]}$ then

10:

$$
x \leftarrow x^{\prime} ; r \leftarrow r^{\prime}
$$

\section{end if}

12: end for

function $\operatorname{LEAPFROG}(x, r, \sigma)$

14: $\quad r^{\prime} \leftarrow r+(\sigma / 2) \nabla_{x} \mathcal{L}(x)$

$x^{\prime} \leftarrow x+\epsilon r^{\prime}$

16: $\quad r^{\prime} \leftarrow r^{\prime}+(\sigma / 2) \nabla_{x} \mathcal{L}\left(x^{\prime}\right)$

return $x,{ }^{\prime} r^{\prime}$

\section{8: end function}

\section{Simulation Details and Results}

We contrasted the statistics of the three samplers for three types of target distribution: a Normal distribution, $\mathcal{N}(1,1)$, a Student's t distribution, $T(1,1, \eta=2)$, and a bimodal distribution that was an equally-weighted mixture of two normal distributions: $\mathcal{N}_{1}(1,1)$ and $\mathcal{N}_{2}(16,1)$. The samplers evaluated were $\mathrm{RWM}, \mathrm{MC}^{3}$, and HMC. We evaluated power-law exponents and sample autocorrelations for MCMC, $\mathrm{MC}^{3}$, and $\mathrm{HMC}$, under different parametrizations, randomly sampling the parametrizations for each algorithm on each run.

For RWM, we varied the proposal width in relation to the width of the target distribution $\sigma_{\text {prop }} / \sigma_{\text {tar }}$. We employed a two-step sampling procedure, first sampling if the proposal width is larger or smaller than the target width with equal probability. Then we sampled $x$ uniformly $x \propto \mathcal{U}(1,10)$, resulting in samples from the range $\left[\sigma_{t a r} / x, x / \sigma_{t a r}\right]$, including both proposal distributions that were significantly larger than the target distribution and those that were much narrower than the target distribution. This was important because the proposal width directly affects the samples' 
autocorrelation: very wide proposal distributions will result in proposals outside of the typical set of the target distribution, producing large numbers of rejections, and thus, since the current state is rarely updated, large autocorrelations. However, this behavior can be subtly different for target distributions that are multimodal or for heavy-tailed distributions. In these cases, occasional large jumps might occur when a proposal into the tails or the other mode is proposed.

For HMC, we kept the step size constant (0.1), as it only affects the integration accuracy. We varied the path length, again sampling with equal probability from $[1 / x, x / 1]$, with $x \propto \mathcal{U}(1,20)$ since small path lengths increase the samples' autocorrelation.

Finally, for $\mathrm{MC}^{3}$, we kept the number of chains fixed (4) but varied the temperature increments between chains, $\left.\delta_{t}=\mathcal{U}(0.5,10)\right)$ and the widths of the proposal distributions as in RWM. Increasing the spacing of temperatures, $\mathrm{MC}^{3}$ can exhibit features less characteristic of RWM: reduced autocorrelation and power-law distributed step sizes.

We sampled starting points randomly for all samplers, $\mathcal{U}(-5,25)$ and simulated 1000 traces with random parametrizations for each target distribution. RWM and $\mathrm{MC}^{3}$ were run for 2000 iterations in each simulation. Since HMC is more sample efficient, but also computationally more costly, we ran HMC simulations for 500 iterations each. Unlike the experimental data, we were not concerned about using upper- or lower-bounds to exclude rest periods or lapses of attention in the simulated data. Thus, we fitted 'pure' power-law exponents using (Alstott \& Bullmore, 2014).

In addition to evaluating distribution tails and the autocorrelation of the samples, we also measured how well the samplers performed in sampling from the target distribution. To do so, we measured the distances between mean embeddings of features of the samples and i.i.d. samples from the target distribution, using Maximum Mean Discrepancy (MMD, for a review, see Muandet, Fukumizu, Sriperumbudur, Schölkopf, et al., 2017). Overall, $\mathrm{MC}^{3}$ produced the lowest MMD values $(M=0.06, S D=0.11)$, followed by MCMC $(M=0.09, S D=0.14)$ and $\mathrm{HMC}(M=0.22, S D=0.25)$. These 
results were consistent across all three target distributions, but most pronounced for the bimodal target $\left(\mathrm{MC}^{3} M_{M M D}=0.1, S D=0.11 ; \mathrm{MCMC} M_{M M D}=0.15, S D=0.11\right.$; HMC $\left.M_{M M D}=0.27, S D=0.09\right)$. Note that these results do not suggest that HMC is an inferior sampling algorithm. On the contrary, HMC algorithms, when carefully tuned to the task at hand, have shown excellent sample efficiency, low autocorrelation, and were able to recover complex distributions. Instead, the scope of this analysis is to show that $\mathrm{MC}^{3}$ achieves characteristic $1 / f$ and power-law statistics, which are usually not desired features of a sampler, under broad parameter initializations and without tuning, while also achieving acceptable distribution recovery. 


\section{References}

Alstott, J., \& Bullmore, D. P. (2014). powerlaw: a Python package for analysis of heavy-tailed distributions. PloS One, $9(1)$.

Altekar, G., Dwarkadas, S., Huelsenbeck, J. P., \& Ronquist, F. (2004). Parallel Metropolis coupled Markov chain Monte Carlo for Bayesian phylogenetic inference. Bioinformatics, $20(3), 407-415$.

Brooks, S., Gelman, A., Jones, G., \& Meng, X.-L. (2011). Handbook of Markov chain Monte Carlo. CRC press.

Chater, N., Tenenbaum, J. B., \& Yuille, A. (2006). Probabilistic models of cognition: Conceptual foundations. Trends in Cognitive Sciences, 10(7), 287-291.

Clauset, A., Shalizi, C. R., \& Newman, M. E. (2009). Power-law distributions in empirical data. SIAM review, 51(4), 661-703.

Gilden, D. L. (1997). Fluctuations in the time required for elementary decisions. Psychological Science, 8(4), 296-301.

Gilden, D. L., Thornton, T., \& Mallon, M. W. (1995). 1/f noise in human cognition. Science, 267(5205), 1837-1839.

Griffiths, T. L., \& Tenenbaum, J. B. (2006). Optimal predictions in everyday cognition. Psychological Science, 17(9), 767-773.

Hills, T. T., Jones, M. N., \& Todd, P. M. (2012). Optimal foraging in semantic memory. Psychological Review, 119(2), 431.

Humphries, N. E., Weimerskirch, H., Queiroz, N., Southall, E. J., \& Sims, D. W. (2012). Foraging success of biological Lévy flights recorded in situ. Proceedings of the National Academy of Sciences, 109(19), 7169-7174.

Jones, M. N., \& Mewhort, D. J. (2007). Representing word meaning and order information in a composite holographic lexicon. Psychological Review, 114(1), 1.

Kahneman, D., Sibony, O., \& Sunstein, C. R. (2021). Noise: A flaw in human judgment. London: William Collins.

MacKay, D. J. (2003). Information theory, inference and learning algorithms. Cambridge University Press. 
Mandelbrot, B. B. (2001). Scaling in financial prices: Iii. Cartoon Brownian motions in multifractal time. Quantitative Finance, 1, 427-440.

Mandelbrot, B. B., \& Van Ness, J. W. (1968). Fractional Brownian motions, fractional noises and applications. SIAM Review, 10(4), 422-437.

Muandet, K., Fukumizu, K., Sriperumbudur, B., Schölkopf, B., et al. (2017). Kernel mean embedding of distributions: A review and beyond. Foundations and Trends in Machine Learning, 10(1-2), 1-141.

Orbán, G., Berkes, P., Fiser, J., \& Lengyel, M. (2016). Neural variability and sampling-based probabilistic representations in the visual cortex. Neuron, 92(2), $530-543$.

Rhodes, T., \& Turvey, M. T. (2007). Human memory retrieval as Lévy foraging. Physica A: Statistical Mechanics and its Applications, 385(1), 255-260.

Troyer, A. K., Moscovitch, M., \& Winocur, G. (1997). Clustering and switching as two components of verbal fluency: evidence from younger and older healthy adults. Neuropsychology, $11(1), 138$.

Viswanathan, G. M., Buldyrev, S. V., Havlin, S., da Luz, M. G. E., Raposo, E. P., \& Stanley, H. E. (1999). Optimizing the success of random searches. Nature, $401(6756), 911-914$.

Zhu, J.-Q., Sanborn, A. N., \& Chater, N. (2020). The Bayesian sampler: Generic Bayesian inference causes incoherence in human probability judgments. Psychological Review, 127(5), 719. 